\title{
Síndrome metabólico y puesto de trabajo
}

\section{Metabolic syndrome and workplace}

\author{
Juan Carlos Palomino Baldeon \\ Medicina Ocupacional y Medio Ambiente. Clínica del Trabajador. Lima, Perú. \\ Medicina Ocupacional y Medio Ambiente. Facultad de Medicina "Alberto Hurtado". \\ Universidad Pervana Cayetano Heredia. Lima. Perú.
}

Gian Carlos Navarro Chumbes

Medicina del Trabajo. Hospital Universitario Ramón y Cajal. Madrid. España.

Recibido: 09-10-10

Aceptado: 25-1 1-10

\section{Correspondencia:}

Gian Carlos Navarro Chumbes

C/ Sierra de Ayllón, 5

19004. Guadalajara. España.

Correo electrónico: gcnavarro@yahoo.com

Resumen

Introducción: El Síndrome Metabólico (SM) aumenta el riesgo de enfermedad cardiovascular y diabetes. Nuestro objetivo es conocer la prevalencia del SM en nuestra población y conocer la diferencia de prevalencias de SM entre trabajadores que trabajan en zona industrial y zona administrativa u oficina, independientemente de la ocupación específica.

Materiales y Métodos: La población fue dividida en zona industrial (Tipo 1) y no industrial u oficina (Tipo 2). Es un estudio Caso Control; la exposición es el trabajo en zona industrial y la enfermedad es la presencia de SM. Se utilizó un modelo multivariado usando la regresión linear.

Resultados: 891 trabajadores fueron evaluados, población Tipo 1 fue 722 (81,03\%) y población Tipo 2 fue 169 (18,97\%). La prevalencia de SM fue 27,83\%. La prevalencia de SM en población Tipo 1 y Tipo 2 fue de 28,94\% y 23,07\% respectivamente, $\mathrm{p}<0,05$. Se encontró $\mathrm{p}<0,01$ para edad, IMC (Índice de Masa Corporal), tensión arterial, glicemia, triglicéridos y HDL. El riesgo relativo del trabajo Tipo 1 para tener SM fue de 1,358 IC (1,19-2,45).

Conclusión: Trabajadores Tipo 1 tienen mayor riesgo de SM debido a una alimentación rica en calorías y menor esfuerzo físico en las actividades de su puesto de trabajo específico.

Med Segur Trab (Internet) 2010; 56 (221): 280-287

Palabras claves: Síndrome Metabólico, Lugar de Trabajo, Trabajador, Productividad.

Abstract

Introduction: The Metabolic Syndrome (MS) raises Diabetes and Cardiovascular disease risks. Our goal is to know MS prevalence in our population and to know the difference of MS prevalence between workers that work in an industrial zone versus administrative or office zone independently of their specific occupation.

Materials and methods: Population was divided in industrial zone (Type 1) and non industrial or office zone (Type 2). It is a Case-Control study; the exposure is the job in industrial zone and the disease is the presence of MS. A multivariate model using lineal regression was used. 
Results: 891 workers were evaluated, Type 1 population was $722(81.03 \%)$ and Type 2 population was 169 (18.97\%). MS prevalence was $27.83 \%$. Type 1 MS prevalence was $28.94 \%$ and Type 2 MS prevalence was $23.07 \%, \mathrm{p}<0.05$. It was found $\mathrm{p}<0.01$ for age, Body mass index (BMI), Arterial Tension, Glycemia, triglycerides and HDL measures. Relative risk of Type 1 job for complaint MS was 1.358 CI (1.19-2.45).

Conclusion: Those Type 1 workers have more risk of MS because of high calories food consumption and less physical effort overcoat in specific job activities.

Med Segur Trab (Internet) 2010; 56 (221): 280-287

Key words: Metabolic Syndrome, Job Placement, Worker, Productivity. 


\section{INTRODUCCIÓN}

El Síndrome Metabólico (SM) es un complejo desorden que afecta a varios sistemas (endocrino, vascular) que conlleva un aumento del riesgo de enfermedad cardiovascular y especialmente de enfermedad coronaria y diabetes ${ }^{1}$. El 3rd. Report of the National Colesterol Education Program - Adult Treatment Panel (ATP-III) lo ha definido como la asociación de múltiples factores de riesgo para el desarrollo de enfermedad cardiovascular y se observa asociado en la mayoría de los pacientes a resistencia insulínica. Puede incluir: trastorno de los lípidos, trastorno del manejo de la glucosa, obesidad e hipertensión en diferentes combinaciones según el criterio de definición empleada ${ }^{2}$.

El aumento progresivo del Síndrome Metabólico hace prever una nueva epidemia mundial de enfermedades cardiovasculares por lo que en la actualidad se considera un importante problema de salud pública ${ }^{3,4}$.

Las medidas de tratamiento y prevención más efectivas del SM radican en tratar o evitar la aparición de sus componentes propiciando estilos de vida saludables. Existe sólida evidencia científica sobre el beneficio de una buena conducta nutricional y física en la mejora de la insulinorresistencia ${ }^{5}$.

Los médicos ocupacionales, sobre todo los que estamos en la Minería Peruana, nos enfrentamos a un paradigma muy antiguo pero que sin embargo puede debilitar cualquier tipo de programa actual de prevención de obesidad o aumento de peso; este paradigma señala que aquellos trabajadores que trabajan en la zona industrial independientemente del tipo de ocupación deben de alimentarse "bien"; entendiéndose esto como mayor cantidad de alimentos en sus comidas y/o mayor número de comidas al día con diferentes presentaciones de acuerdo a la región.

El objetivo de este estudio es conocer la prevalencia del SM en nuestra población y conocer la diferencia de prevalencias de SM entre trabajadores que trabajan en zona industrial versus zona administrativa independientemente de su ocupación específica.

\section{MATERIALES Y MÉTODOS}

El estudio fue realizado durante el año 2006 en el área productiva de una minera del sur del Perú del rubro de gran Minería que se encuentra aproximadamente a $3.300 \mathrm{msnm}$, siendo un estudio tipo caso control. Se incluyó toda la población de trabajadores mayores de 40 años, dado que el examen anual incluye medida de lípidos en los trabajadores a partir de esta edad, determinándose como exposición el trabajo en zona industrial y como enfermedad la presencia de SM definido según los criterios de ATP III. El resto de la población trabajadora que pasó su revisión médica anual fue considerada como controles.

Se evaluó una población total de 891 trabajadores, entre administrativos y operadores, que acudieron a la evaluación médica anual. Una característica importante de nuestra población es que su trabajo para una empresa minera privada les garantiza un buen ingreso económico estable. Se respetó en todo momento la confidencialidad de los participantes involucrados en el estudio.

\section{Criterios de inclusión}

Todo trabajador que haya pasado su evaluación médica anual del año 2006.

Edad igual o mayor a 40 años.

\section{Criterios de exclusión}

Trabajadores que no hayan pasado la evaluación médica anual completa, que les falten evaluaciones clínicas o resultados de exámenes de laboratorio. 


\section{METODOLOGÍA}

A partir de la revisión médica anual del año 2006 que pasaron todos los trabajadores de la minera, se elaboró una base de datos electrónica que consignó las variables de edad, sexo, puesto de trabajo, tiempo de trabajo en la empresa, talla, peso, circunferencia de cintura, circunferencia de cadera (C), relación cintura-cadera (CC), índice de masa corporal (IMC), presión sistólica (PS), presión diastólica (PD), lectura de presión arterial e hipertensión arterial (HTA), diabetes mellitus (DM), además se obtuvo valor de glicemia, de colesterol y triglicéridos.

Para definir la presencia del SM se utilizaron los criterios de ATP III corregidos.

- Obesidad abdominal: Definida como circunferencia de la cintura $>102 \mathrm{~cm}$ en los hombres y de $88 \mathrm{~cm}$ en las mujeres.

- Triglicéridos altos: Definidos si el valor es $\geq 150 \mathrm{mg} / \mathrm{dl}$.

- Colesterol HDL bajo: Si el valor es $<40 \mathrm{mg} / \mathrm{dl}$ en hombres y $<50 \mathrm{mg} / \mathrm{dl}$ en mujeres.

- Hipertensión arterial: Si el valor es $\geq$ a $130 / \geq 85 \mathrm{mmHg}$.

- Hiperglucemia en ayunas: Valor $\geq 100 \mathrm{mg} / \mathrm{dl}$.

Se realizó el diagnóstico de síndrome metabólico cuando se encontraban presentes tres o más de los factores de riesgo.

De acuerdo al puesto de trabajo dividimos nuestra población en población que trabaja en zona industrial (Tipo 1) y no industrial (Tipo 2) de acuerdo a la evaluación del puesto realizada por el médico ocupacional o médico del trabajo durante la evaluación médica anual.

La talla fue medida en metros, estando el individuo descalzo y en posición supina, utilizando un tallímetro calibrado con una precisión de $\pm 0,5 \mathrm{~cm}$. El peso fue medido en kilogramos, con el individuo desnudo y descalzo, con balanza digital calibrada con una precisión de 1 gramo. Ambas mediciones fueron realizadas por los autores.

Los diagnósticos de HTA y DM fueron obtenidos de la Historia Clínica Electrónica de cada paciente.

El IMC fue calculado como peso (kilogramos) dividido por la talla al cuadrado (metros cuadrados). El sobrepeso y la obesidad fueron definidos por las recomendaciones de la OMS: IMC $<24,9 \mathrm{~kg} / \mathrm{m} 2$ : normales; IMC entre 25 y 29,9 kg/m²: sobrepeso; e IMC $\geq 30 \mathrm{~kg} / \mathrm{m}^{2}$ : obesos. La circunferencia de la cintura fue medida con el individuo en espiración en el punto medio entre el reborde costal y la cresta iliaca que presenta el menor diámetro. La circunferencia de la cadera se realiza con el individuo de pie, con los glúteos relajados y los pies juntos, a nivel de los trocánteres mayores, que en general coinciden con la sínfisis pubiana.

\section{ANÁLISIS ESTADÍSTICO}

Todos los datos fueron ingresados en una hoja de cálculos de Excel versión 2000, para luego ser trasladados y procesados con Software SPSS 12.0.

Las variables cuantitativas fueron categorizadas para convertirlas en variables dicotómicas. Se realizaron las pruebas de la t de Student y de la $\chi^{2}$ para valorar las diferencias estadísticas entre las características de los participantes.

Las pruebas estadísticas fueron de 2 colas, el nivel de confianza fue de $95 \%$ y se consideraron estadísticamente significativos los valores de $\mathrm{p} \leq 0,05$.

Las variables con valores de $\mathrm{p}<0,01$ en el análisis univariado fueron examinadas en un modelo multivariado usando la regresión linear. 


\section{RESULTADOS}

El número de participantes fue de 897 trabajadores, de los cuales fueron excluidos 6 debido a que cumplieron parcialmente con la revisión médica faltando información sobre los mismos.

El número de participantes varones y mujeres fue de $848(95,17 \%)$ y $43(4,83 \%)$ respectivamente.

El número de participantes catalogados como Tipo 1 y Tipo 2 fue de $722(81,03 \%)$ y de 169 (18,97\%), respectivamente. La prevalencia de SM de toda la población fue de $27,83 \%$. De acuerdo al tipo de población se encontró una prevalencia de SM de $28,94 \%$ y un $23,07 \%$ para la población tipo 1 y tipo 2 , respectivamente $(\mathrm{p}<0,05)$. Cuando evaluamos cuáles serían los criterios encontrados más frecuentemente entre aquellos trabajadores con SM se obtuvo que los criterios de mayor porcentaje en orden decreciente fueron: HDL bajo, 81,05\%; triglicéridos elevados, 48,39\%; circunferencia abdominal, 45,97\%; glicemia elevada, 39,1\%, y tensión arterial elevada, 23,79\%.

Cuando se evaluó la presencia o no de SM por grupo etáreo encontramos que el mayor grupo se encontraba entre los 41-60 años ( $\mathrm{p}<0,05)$. No se encontraron diferencias con el tiempo de trabajo ( $p>0,05$ ). Cuando se evaluó la presencia o no de SM de acuerdo al IMC podemos apreciar que los grupos predominantes son los que se encuentran entre 25-29,9 años y 30-34,9 años ( $\mathrm{p}<0,05)$.

Al evaluar la presencia o no de SM de acuerdo al diagnóstico de HTA, encontramos que de los que tienen SM el 28\% tiene el diagnóstico de HTA versus el $10 \%$ que no lo es $(\mathrm{p}<0,05)$ y al evaluar la presencia o no de SM de acuerdo a la presencia de diabetes mellitus (DBM), encontramos que de los que tienen SM el 11\% tiene DBM versus el 1,05\% que no lo es $(\mathrm{p}<0,05)$.

Al evaluar la presencia o no de SM de acuerdo a la presencia de hipertrigliceridemia (TGS), encontramos que de los que tienen SM el 89\% tiene TGS versus el 48,7\% que no lo tiene $(\mathrm{p}<0,05)$.

Al realizar el análisis de regresión, siendo la variable dependiente SM ATP III, se encontró que fue significativo ( $\mathrm{p}<0,01$ ) para las variables edad, IMC, medida de presión arterial, glicemia, TGS y HDL, lo cual va de acuerdo con la definición de SM ATP III (tabla 1).

TABLA 1. Características generales del total de la población de acuerdo a la presencia de Sindrome Metabólico

\begin{tabular}{|c|c|c|c|c|}
\hline & Total $(n=891)$ & $\mathbf{p}^{*}$ & $\operatorname{Sin} S M(n=643)$ & Con $\operatorname{SM}(n=248)$ \\
\hline Edad (años), media $\pm \mathrm{DE}$ & $50,83 \pm 8,07$ & $<0,05$ & $50,21 \pm 4,24$ & $52,46 \pm 2,82$ \\
\hline Tiempo de servicio (meses), media $\pm \mathrm{DE}$ & $25,04 \pm 12,01$ & $>0,05$ & $24,49 \pm 7,07$ & $26,46 \pm 0,71$ \\
\hline $\mathrm{IMC}$, media $\pm \mathrm{DE}$ & $28,34 \pm 3,23$ & $<0,05$ & $27,63 \pm 2,67$ & $30,20 \pm 0,65$ \\
\hline Cintura $(\mathrm{cm})$, media $\pm \mathrm{DE}$ & $96,17 \pm 8,27$ & $<0,05$ & $94,15 \pm 10,61$ & $101,40 \pm 0,00$ \\
\hline Cadera $(\mathrm{cm})$, media $\pm \mathrm{DE}$ & $97,25 \pm 5,67$ & $<0,05$ & $96,29 \pm 4,24$ & $99,71 \pm 2,83$ \\
\hline Glicemia basal $(\mathrm{mg} / \mathrm{dl})$, media $\pm \mathrm{DE}$ & $88,17 \pm 20,63$ & $<0,05$ & $83,70 \pm 103,24$ & $99,74 \pm 194$ \\
\hline Colesterol total $(\mathrm{mg} / \mathrm{dl})$, media $\pm \mathrm{DE}$ & $204,64 \pm 9,19$ & $>0,05$ & $202,83 \pm 41,72$ & $209,35 \pm 6,36$ \\
\hline Colesterol HDL $(\mathrm{mg} / \mathrm{dl})$, media $\pm \mathrm{DE}$ & $42,05 \pm 9,06$ & $<0,05$ & $44,22 \pm 4,95$ & $36,40 \pm 6,36$ \\
\hline Triglicéridos $(\mathrm{mg} / \mathrm{dl})$, media $\pm \mathrm{DE}$ & $193,16 \pm 105,75$ & $<0,05$ & $169,23 \pm 25,46$ & $255,00 \pm 103,94$ \\
\hline $\begin{array}{l}\text { Presión arterial sistólica }(\mathrm{mmHg}) \\
\text { media } \pm \mathrm{DE}\end{array}$ & $123,69 \pm 14,64$ & $<0,05$ & $120,21 \pm 0,00$ & $133,00 \pm 7,10$ \\
\hline $\begin{array}{l}\text { Presión arterial diastólica }(\mathrm{mmHg}) \\
\text { media } \pm \mathrm{DE}\end{array}$ & $80,71 \pm 9,50$ & $<0,05$ & $78,87 \pm 7,07$ & $85,00 \pm 0,00$ \\
\hline
\end{tabular}

Cuando se realiza el análisis de regresión para hallar el riesgo del trabajo tipo 1 para realizar SM encontramos un Odds ratio de 1,35 (OR $>1$ ) con un intervalo de confianza válido (tabla 2). 
TABLA 2. Odds ratio según tipo de trabajo

\begin{tabular}{lcccc}
\hline & Sin SM $(\mathbf{n}=\mathbf{6 4 3})$ & Con SM $(\mathbf{n}=\mathbf{2 4 8})$ & OR & IC \\
\hline Tipo $2, \%$ & 20,22 & 15,73 & 0,73 & $0,23-0,89$ \\
Tipo 1, \% & 79,78 & 84,27 & 1,358 & $1,19-2,45$ \\
\hline \multicolumn{4}{l}{ SM: Síndrome Metabólico; OR: Odds ratio; IC: intervalo de confianza. } \\
\hline
\end{tabular}

\section{DISCUSIÓN}

Durante la práctica diaria los médicos ocupacionales o médicos del trabajo nos enfrentamos al desafío de liderar los programas de prevención de enfermedades ocupacionales y enfermedades comunes, siendo en la actualidad el aumento de peso uno de los mayores problemas de salud en nuestras poblaciones y en el mundo actual ${ }^{6}$.

El SM es una condición que se caracteriza por presentar resistencia a la insulina, la cual estaría determinando la dislipidemia ateroesclerótica; la hiperinsulinemia; los niveles elevados de glicemia, la presión arterial elevada, un estado protrombótico e inflamatorio y sobre todo la obesidad abdominal o central. Los pacientes que sean positivos para dicho síndrome tendrían más riesgo durante toda su vida para enfermedades cardiovasculares y diabetes por lo que es importante detectarlos ${ }^{7,8}$.

En una población trabajadora todas las enfermedades afectan a la productividad de la empresa tanto de forma directa (costos médicos por enfermedad) como indirecta (absentismo médico, presentismo, etc.) 9; es por esto que se deben realizar estrategias para la prevención de diversas enfermedades y sobre todo de la aparición del SM.

El primer paso para la ideación de estrategias para estos programas se inicia conociendo las características e idiosincrasia de nuestra población trabajadora y de sus familias ${ }^{10}$. La prevalencia del SM fluctúa ampliamente dependiendo de la población estudiada, por ejemplo, en la National Colesterol Education Program Treatment Panel III (ATP III) de acuerdo a dichos criterios, encuentran en la población americana una prevalencia de $24 \%$ en adultos ${ }^{11}{ }^{12}$, en adultos guatemaltecos varía desde 7 al $28 \%$, de acuerdo de que se tratasen de agricultores, población rural y urbana ${ }^{13}$, en el Perú, Guarnizo-Poma et al, en el 2006, encuentran un 31,7\% en una zona agroganadera y de $22,1 \%$ en una zona pesquera de Lambayeque (Perú) ${ }^{14}$. Nosotros hemos encontrado en toda la población estudiada una prevalencia de $27,83 \%$; dicho valor se encontraría dentro de lo señalado por la literatura para población general, sin embargo, cuando se evalúan las prevalencias en población laboral se encuentran investigaciones que nos indican que el SM fluctúa entre 7 a 16\% en España y Países del Oriente ${ }^{15,16,17}$ las cuales están por debajo de nuestra prevalencia encontrada; estas menores prevalencias se deben probablemente porque son poblaciones laborales más jóvenes que la nuestra y también por el efecto del trabajador sano ${ }^{18}$. En nuestra población hallamos un Odds Ratio de 1,35 de desarrollar SM en aquellos trabajadores de la población tipo 1, lo cual coincide con el estudio MESYAS de España ${ }^{16}$.

En todas las empresas hay una diversidad de puestos de trabajos, pudiéndose dividir en dos grandes grupos: trabajo de oficina y trabajo de campo; estos últimos llamados puestos de trabajos de zona industrial donde generalmente los trabajadores realizan tareas que involucran carga de peso y esfuerzo físico durante el turno de trabajo ${ }^{19}$. Las investigaciones encuentran que la actividad física beneficia la salud de las personas ${ }^{20,21}$; por lo que en teoría aquellos trabajadores con mayor actividad física de acuerdo a la ocupación podrían tener menores niveles de sobrepeso y obesidad dado el mayor gasto energético en el trabajo, sin embargo, esto dependería también de la alimentación ${ }^{22,23,24 .}$ Nosotros conocemos de la práctica diaria que entre los trabajadores de zona industrial y sus familias se tiene la concepción de que deben de alimentarse "bien", de tener una "mayor" alimentación comparado con el trabajador de oficina, se debe de notar que la concepción no es de mayor calidad sino de mayor cantidad, casi nunca realizando la distinción de acuerdo al gasto energético de acuerdo a su actividad física en el trabajo, por lo que muchos trabajadores que trabajan en zona industrial en ocupaciones que no 
demandan actividad física importante se alimentan con esta concepción; siendo estos los trabajadores que producen los mayores problemas de no adhesión y problemas de seguimiento a los programas de prevención de obesidad ${ }^{25}$, generalmente las normas no se ocupan del tema de la alimentación de acuerdo al gasto energético del tipo de trabajo, sólo algunos reportes evalúan este tema ${ }^{26}$.

Dependiendo de la zona de nuestro país existe predominancia de determinados alimentos; así en el estudio de Guarnizo-Poma et al comparar entre una población de sierra y costa encuentran que en la población andina predominan los alimentos ricos en carbohidratos y grasas ${ }^{14}$; estos alimentos están relacionados al aumento de peso y SM ${ }^{22}$.

En la población estudiada, cuando comparamos ambos grupos, podemos señalar que la población Tipo 1 tiene niveles mayores de SM siendo estadísticamente significativa; esta diferencia podría ser explicada porque la población Tipo 1 no tiene una alimentación saludable, lo cual se refleja cuando encontramos que el HDL bajo y triglicéridos elevados son los elementos más frecuentemente encontrados entre aquellos con SM, además la población Tipo 1 tampoco realiza toda la actividad física que se pensaría dado que debemos de tener en cuenta la progresión tecnológica en las grandes empresas donde se señala la evolución del obrero desde los artesanales (típicos del pasado) y los científicos (típicos de la actualidad y sobre todo del futuro) cuentan con un trabajo con carácter puramente instrumental y automatizado, llamando a los obreros manuales de industrias automatizadas un obrero poco manual ${ }^{16}$. Por otro lado, la población tipo 2 tendrían más cuidado en la manutención de la salud; es decir se preocupan más de ejercicios, rutinas y alimentación saludable dado que son conscientes de que su actividad física durante las horas de trabajo es mínima, tal como se encuentra en una población similar en el estudio MESYAS ${ }^{16}$, donde encontraron que los trabajadores que realizaban trabajos de oficina tenían menor prevalencia de SM, explicando que esto se debería porque dicha población tenía mayor nivel de instrucción con la consiguiente mayor preocupación por la conservación de su salud; aunque nosotros no tenemos la información sobre el grado de instrucción, podemos inferir que en nuestra población tipo 2 es mayor el grado de instrucción dado que los puestos de trabajo en su mayoría son de carreras universitarias (ingenieros, abogados, médicos, etc.) mientras que en la población tipo 1 son más frecuentes las carreras técnicas (soldador, mecánico, operador de equipos, etc.) ${ }^{27,28}$. Es decir, en la zona industrial hay una gran estratificación de ocupaciones que dependen de sus niveles de calificación y responsabilidad con las consiguientes diferencias de gasto energético ${ }^{26}$. Finalmente pensamos que en la actualidad las poblaciones tipo 1 no solamente no tienen una alimentación saludable sino que su gasto energético en el trabajo es menor que en el pasado sobre todo en la categoría de gran minería.

Conociendo que hay un incremento mundial de la prevalencia de SM y que nuestro objetivo es la prevención de esta condición concluimos en nuestra población que:

- Los trabajadores mineros que trabajan en zona industrial (tipo 1) tienen mayor riesgo de desarrollar SM.

- Se deberían de realizar estudios que comparen grupos de trabajadores de acuerdo a su gasto energético o similar para poder conocer realmente el efecto protector de la actividad física durante el trabajo.

- Los médicos ocupacionales o médicos del trabajo de las mineras deben planificar sus programas de salud tomando en cuenta el gasto energético por puestos o áreas de trabajo y la educación nutricional de cada trabajador. 


\section{REFERENCIAS BIBLIOGRÁFICAS}

1. Roche HM; Phillips C; Gibney M. The metabolic syndrome: the crossroads of diet and genetics. Proccedings of the Nutrition Society. 2005; 64: 371-77.

2. Third report of the National Cholesterol Education Program (NCEP). Expert Panel on the detection, evaluation, and treatment of high blood cholesterol in adults (Adult Treatment panel III). Executive summary. NIH Publication 01-3670; May 2001.

3. Isomaa B, Almgren P, Tuomi T, Forsen B, Lahti K, Nissen M, Taskinen MR, Groop L. Cardiovascular morbidity and mortality associated with the metabolic syndrome. Diabetes Care. 2001 Apr; 24(4):683-9.

4. Muredach P. Reilly and Daniel J. Rader. The Metabolic Syndrome: More than the Sum of Its Parts? Circulation 2003; 108; 1546-1551

5. Burrowes J.D. Metabolic Syndrome. Nutrition Today 2006; 41(3): 131-137.

6. Serra C. Company A. Vigilancia de la Salud. En Salud Laboral Conceptos y técnicas para la prevención de riesgos laborales. 2007, Editorial Masson, 3. ${ }^{\text {a }}$ Edición.

7. Coleman RJ, Stevens RJ, Retnakaran R, Framingham, SCORE, and DECODE Risk Equations do not provide reliable Cardiovascular Risk Estimates in Type 2 diabetes. Diabetes Care. 2007; 30(5) 1292-93.

8. Grundy Scott. Metabolic Syndrome: A Multiplex Cardiovascular Risk factor. J Clin Endocrinol Metab 2007; 92:399-404.

9. Allen, Harris PhD. Using Routinely Collected Data to Augment the Management of Health and Productivity Loss. JOEM: 2008. Vol 50(6) June, pp. 615-632.

10. Cox Carolyn. Designing Health Promotion Programs in Worksite Health Promotion Manual. 2003. Edit Human Kinetics IV Title.

11. Ford ES, Giles WH. Dietz WH. Prevalence of the metabolic Syndrome among US Adults. JAMA 2002; $287: 356$ - 359.

12. Ford ES, Giles WH. Dietz WH. A comparison of the prevalence of the metabolic syndrome using two proposed definitions. Diabetes Care 2003; 26: 575-581.

13. Gregory Co, Dai J, Ramirez-Zea M, Stein AD. Occupation is more important than rural or urban residence in explaining the prevalence of Metabolic and Cardiovascular disease risk en Guatemalan adults. J Nutr. 2007; 137 (5): 1314 -9.

14. Guarnizo MF, Loayza G, Calvay M, Ynami MZ, Lázaro H. Síndrome Metabólico en una población pesquera y otra agroganadera de la costa del Perú. Rev. Soc. Per. Med. Inter. 2006; 19(1).

15. Cordero A, Alegría E, León M. Prevalencia de síndrome metabólico. Rev Esp Cardiol Supl. 2005; 5:11D-5D.

16. Alegría E., Cordero A, Laclaustra M, et al. Prevalencia del síndrome metabólico en población laboral española: registro MESYAS. Rev Esp Cardiol. 2005; 58(7):797-806.

17. Shiwaku K, Nogi A, Kitajima K, Anuurad E. Prevalence of the Metabolic Síndrome using the Modified ATP III definitions for Workers in Japan , Korea and Mongolia. J Occup Health 2005; 47: 126-135.

18. Puig T, Varas C, Pérez I, Abadal LT y Balaguer I. Patrones de mortalidad en una cohorte de trabajadores seguida durante 28 años: estudio Manresa. Rev Esp Cardiol 2004; 57(10):924-30.

19. Allen H, Woock C, Barrington L, Bunn W. Age, overtime, and employee health, safety and productivity outcomes: a case study. JOEM. 2008 Aug; 50(8):873-94.

20. Darren E.R. Warburton, Crystal Whitney Nicol, Shannon S.D. Bredin. Health benefits of physical activity: the evidence Review CMAJ 2006; 174(6):801-9.

21. Bensimhon, Daniel R. MD; Kraus, William E. MD; Donahue, Mark P. MD. Obesity and physical activity: A review. American Heart Journal. 2006; 151(3):598-603.

22. Racette SB, Weiss EP, Schechtman KB, Steger-May K, Villareal DT, Obert KA, Holloszy JO. Influence of weekend lifestyle patterns on body weight. Obesity (Silver Spring). 2008 Aug; 16(8):1826-30.

23. Lee KW, Lip GY.The role of omega-3 fatty acids in the secondary prevention of cardiovascular disease. QJM. 2003 Jul; 96(7):465-80

24. Barrett PH, Watts GF. Kinetic studies of lipoprotein metabolism in the metabolic syndrome including effects of nutritional interventions. Curr Opin Lipidol. 2003 Feb; 14(1):61-8.

25. Toole T, Thorn JE, Panton LB, Kingsley D, Haymes EM. Effects of a 12-month pedometer walking program on gait, body mass index, and lower extremity function in obese women. Percept Mot Skills. 2007 Feb; 104(1):212-20.

26. Condiciones de Trabajo, Seguridad y Salud en la Minería del Perú. Oficina Internacional del Trabajo. OIT. 2002.

27. Santos A.C, Ebrahim S, Barrios H. Gender, socio-economic status and metabolic syndrome in middle- aged and old adults. BMC Public Health. 2008; 8:62.

28. Chandola T, Brunner E, Marmot M. Chronic stress at work and the metabolic syndrome: prospective study. BMJ 2006; 332;521-525. 